PROCEEDINGS OF THE

AMERICAN MATHEMATICAL SOCIETY

Volume 130, Number 2, Pages 483-487

S 0002-9939(01)06063-4

Article electronically published on May 25, 2001

\title{
ITERATED FUNCTION SYSTEMS OF FINITE TYPE AND THE WEAK SEPARATION PROPERTY
}

\author{
NHU NGUYEN
}

(Communicated by Christopher D. Sogge)

\begin{abstract}
We prove that any iterated function system of finite type possesses the weak separation property.
\end{abstract}

\section{INTRODUCTION}

Let $\left\{f_{1}, \ldots, f_{m}\right\}$ be an iterated function system (IFS) on $\mathbb{R}^{d}$ defined by

$$
f_{i}(x)=\rho_{i} M_{i} x+b_{i}, i=1, \ldots, m,
$$

where $0<\rho_{i}<1, b_{i} \in \mathbb{R}^{d}$ and $M_{i}$ is a $d \times d$ orthogonal matrix.

Let $E$ denote the attractor of the IFS (1.1), i.e., the unique compact set $E \subset \mathbb{R}^{d}$ satisfies the equation

$$
E=\bigcup_{i=1}^{m} f_{i}(E)
$$

The problem of calculating the Hausdorff dimension of the attractor of an IFS is one of the most interesting questions in fractal geometry. In general it is quite difficult to calculate the Hausdorff dimension of an attractor. However, in some special situations the Hausdorff dimension of an attractor can be rigorously calculated. These special circumstances include:

1. any IFS satisfying the open set condition (the OSC); see $[\mathrm{H}][\mathrm{F}]$;

2. any IFS of finite type; see $[\mathrm{L}] \mathrm{NW}$.

The Hausdorff dimension of the attractor of an IFS possessing the weak separation property (the WSP) has been investigated in $\overline{\mathrm{LN}}$ and $\mathrm{Z}]$.

In this note we establish a relation between the above conditions. Our result shows that the finite type condition implies the WSP.

Received by the editors February 5, 2000 and, in revised form, July 1, 2000.

1991 Mathematics Subject Classification. Primary 28A78; Secondary 28A80.

Key words and phrases. Hausdorff dimension, open set condition, weak separation property, finite type condition, iterated function system.

This author has previously published as Nguyen To Nhu.

(C)2001 American Mathematical Society 


\section{THE FINITE TYPE CONDITION AND THE WEAK SEPARATION PROPERTY}

Recall that the IFS (1.1) satisfies the open set condition (the OSC) (see $[\mathrm{H}, \mathrm{F}$ ) if there exists an open set $O \subset \mathbb{R}^{d}$ such that

$$
O \supset \bigcup_{i=1}^{m} f_{i}(O) \text { with this union disjoint. }
$$

To describe the WSP and the finite type of (1.1) we need some additional notation. For the IFS given by $(1.1)$, let $\Omega=\{1, \ldots, m\}$, let $\Omega^{k}$ denote the set of all words of length $k$ in $\Omega$, and let $\Omega^{*}=\bigcup_{k=1}^{\infty} \Omega^{k}$ denote the set of all finite words in $\Omega$. For $\mathbf{i} \in \Omega^{k}, \mathbf{j} \in \Omega^{n}$, let $\mathbf{i j} \in \Omega^{k+n}$ denote the concatenation of $\mathbf{i}$ and $\mathbf{j}$. For $\mathbf{i}=\left(i_{1}, \ldots, i_{k}\right) \in \Omega^{k}$ we denote

$$
f_{\mathbf{i}}=f_{i_{1}} \ldots f_{i_{k}}, \quad \rho_{\mathbf{i}}=\rho_{i_{1}} \ldots \rho_{i_{k}}, \quad M_{\mathbf{i}}=M_{i_{1}} \ldots M_{i_{k}} .
$$

Let $\rho=\min \left\{\rho_{i}\right\}$, and for $k=1,2, \ldots$ we define

$$
\Lambda_{k}=\left\{\mathbf{i} \in \Omega^{*}: \rho_{\mathbf{i}} \leq \rho^{k}<\rho_{\mathbf{j}} \text { for any proper subset } \mathbf{j} \text { of } \mathbf{i}\right\},
$$

$$
\Gamma_{k}=\left\{\left(\rho_{\mathbf{i}} M_{\mathbf{i}}, f_{\mathbf{i}}(0), k\right): \mathbf{i} \in \Lambda_{k}\right\} \text { and } \Gamma=\bigcup_{k=1}^{\infty} \Gamma_{k}
$$

Observe that in general $\Lambda_{k}$ is not in one-to-one correspondence with $\Gamma_{k}$. For instance:

Example. Let $f_{i}: \mathbb{R} \rightarrow \mathbb{R}, i=1,2,3$, be defined by

$$
f_{1}(x)=\frac{1}{3} x, \quad f_{2}(x)=\frac{1}{3} x+1, \quad f_{3}(x)=\frac{1}{9} x+\frac{1}{3} .
$$

Then $\rho_{1}=\rho_{2}=1 / 3, \rho_{3}=\rho=1 / 9$ and

$$
\Lambda_{1}=\{(1,1),(1,2),(2,1),(2,2),(3)\} .
$$

Clearly $\mathbf{i}=(1,2) \in \Lambda_{1}, \mathbf{j}=(3) \in \Lambda_{1}$ and $\mathbf{i} \neq \mathbf{j}$, but $\left(\rho_{\mathbf{i}} M_{\mathbf{i}}, f_{\mathbf{i}}(0), 1\right)=\left(\rho_{\mathbf{j}} M_{\mathbf{j}}, f_{\mathbf{j}}(0), 1\right)$ $\in \Gamma_{1}$.

Now for $\mathbf{u}=\left(\rho_{\mathbf{i}} M_{\mathbf{i}}, f_{\mathbf{i}}(0), k\right) \in \Gamma_{k}$ we define the contraction $f_{\mathbf{u}}: \mathbb{R}^{d} \rightarrow \mathbb{R}^{d}$ by

$$
f_{\mathbf{u}}(x)=\rho_{\mathbf{i}} M_{\mathbf{i}} x+f_{\mathbf{i}}(0) \text { for } x \in \mathbb{R}^{d} .
$$

Let $O$ be an open bounded invariant subset of $\mathbb{R}^{d}$, i.e., $f_{i}(O) \subset O$ for all $i=1, \ldots, m$. For $\mathbf{u} \in \Gamma_{k}$ we denote

$$
O(\mathbf{u})=\left\{\mathbf{v} \in \Gamma_{k}: f_{\mathbf{v}}(O) \cap f_{\mathbf{u}}(O) \neq \emptyset\right\},
$$

and call $O(\mathbf{u})$ the $O$-neighborhood of $\mathbf{u}$. For $\mathbf{u} \in \Gamma_{k}, \mathbf{v} \in \Gamma_{n}$ we say that $O(\mathbf{u})$ and $O(\mathbf{v})$ are equivalent, denoted $O(\mathbf{u}) \equiv O(\mathbf{v})$, if there is a similarity map $\tau(x)=$ $\rho^{n-k} M x+b$, where $b \in \mathbb{R}^{d}$ and $M$ is a $d \times d$ orthogonal matrix, such that

$$
f_{\mathbf{v}}=\tau \circ f_{\mathbf{u}} \text { and }\left\{f_{\mathbf{v}^{\prime}}: \mathbf{v}^{\prime} \in O(\mathbf{v})\right\}=\left\{\tau \circ f_{\mathbf{u}^{\prime}}: \mathbf{u}^{\prime} \in O(\mathbf{u})\right\} .
$$

It is easy to see that $\equiv$ is an equivalence relation. Let $[O(\mathbf{u})]$ denote the equivalence class of $O(\mathbf{u})$. From $(2.6)$ we get

$$
\text { If } O(\mathbf{u}) \equiv O(\mathbf{v}) \text {, then } \# O(\mathbf{u})=\# O(\mathbf{v}) \text {. }
$$

Definition $1([\mathrm{NW}])$. The IFS (1.1) is of finite type if there exists a bounded invariant open set $O \subset \mathbb{R}^{d}$ such that $\{[O(\mathbf{u})]: \mathbf{u} \in \Gamma\}$ (where $\Gamma$ is defined by (2.4)) is a finite set. The set $O$ is called a finite type condition set. 
The following definition was given in [LN].

Definition 2. The IFS (1.1) possesses the weak separation property (the WSP) if there exists an $\ell \in \mathbb{N}$ such that for any $\mathbf{i} \in \Omega^{*}$ and every $k \geq 1$, every $\rho^{k}$-ball contains at most $\ell$ distinct points $f_{\mathbf{j i}}(0)$ for $\mathbf{j} \in \Lambda_{k}$. (Observe that $f_{\mathbf{j i}}(0)$ can be repeated, i.e., $f_{\mathbf{j i}}(0)=f_{\mathbf{j}^{\prime} \mathbf{i}}(0)$ with $\mathbf{j} \neq \mathbf{j}^{\prime}$. $)$

In [LN] it was shown that any IFS satisfying the OSC possesses the WSP; however the converse is not true. No similar relations between the finite type condition and the WSP have been established. In [NW] it was conjectured that an IFS of finite type must possess the WSP. We prove that the conjecture is true.

Theorem. If the IFS (1.1) is of finite type, then it possesses the WSP.

As mentioned in [NW] our theorem shows that Theorems 1.2 and 1.3 of $[\mathrm{NW}]$ follow from the earlier results of Zerner; see [Z] Corollary and Theorem 3.

\section{Proof of the Theorem}

Assume that the IFS is of finite type and let $O$ denote a finite type condition set. Without loss of generality we may assume that

$$
\operatorname{diam} O \leq 1 \text { and } \sup \left\{\left\|f_{\mathbf{i}}(0)\right\|: \mathbf{i} \in \Omega^{*}\right\} \leq 1 .
$$

Lemma 1. There exist $x_{0} \in O$ and $\delta>0$ such that for any $\mathbf{u}=\left(\rho_{\mathbf{i}} M_{\mathbf{i}}, f_{\mathbf{i}}(0), k\right) \in$ $\Gamma_{k}$, the set $f_{\mathbf{u}}(O)$ contains the $\delta \rho^{k}$-ball $B\left(f_{\mathbf{i}}\left(x_{0}\right), \delta \rho^{k}\right)$.

Proof. Take $x_{0} \in O$ and let $\delta>0$ such that $B\left(x_{0}, \rho^{-1} \delta\right) \subset O$. We claim that for any $\mathbf{u}=\left(\rho_{\mathbf{i}} M_{\mathbf{i}}, f_{\mathbf{i}}(0), k\right)$ we have $B\left(f_{\mathbf{i}}\left(x_{0}\right), \delta \rho^{k}\right) \subset f_{\mathbf{u}}\left(B\left(x_{0}, \rho^{-1} \delta\right)\right) \subset f_{\mathbf{u}}(O)$.

In fact, observe that for $\mathbf{i}=\left(i_{1}, \ldots, i_{k}\right) \in \Omega^{k}$ the orthogonal matrix $M_{\mathbf{i}}$ given by (2.2) induces an isometry (also denoted by $M_{\mathbf{i}}$ ) from $\mathbb{R}^{d}$ into itself. Thus $M_{\mathbf{i}}\left(B\left(x_{0}, r\right)\right)=B\left(M_{\mathbf{i}}\left(x_{0}\right), r\right)$ for any ball $B\left(x_{0}, r\right)$. Therefore $\rho_{\mathbf{i}} M_{\mathbf{i}}\left(B\left(x_{0}, r\right)\right)=$ $B\left(\rho_{\mathbf{i}} M_{\mathbf{i}}\left(x_{0}\right), \rho_{\mathbf{i}} r\right)$. Observe that for $\mathbf{u}=\left(\rho_{\mathbf{i}} M_{\mathbf{i}}, f_{\mathbf{i}}(0), k\right) \in \Gamma_{k}$ we have

$$
f_{\mathbf{u}}(x)=\rho_{\mathbf{i}} M_{\mathbf{i}}(x)+f_{\mathbf{i}}(0) \text { for every } x \in \mathbb{R}^{d} .
$$

It follows that

$$
B\left(f_{\mathbf{i}}\left(x_{0}\right), \rho^{-1} \delta \rho_{\mathbf{i}}\right)=f_{\mathbf{u}}\left(B\left(x_{0}, \rho^{-1} \delta\right)\right) \subset f_{\mathbf{u}}(O) .
$$

From (2.3) we get

$$
\rho_{\mathbf{i}} \leq \rho^{k}<\rho^{-1} \rho_{\mathbf{i}} \text { for any } \mathbf{i} \in \Lambda_{k} .
$$

Consequently

$$
B\left(f_{\mathbf{i}}\left(x_{0}\right), \delta \rho^{k}\right) \subset B\left(f_{\mathbf{i}}\left(x_{0}\right), \rho^{-1} \delta \rho_{\mathbf{i}}\right) \subset f_{\mathbf{u}}(O) .
$$

The lemma is proved.

For any $\mathbf{i} \in \Omega^{*}$ and every $k \geq 1$, let $B\left(\rho^{k}\right)$ be a ball of radius $\rho^{k}$, and let

$$
\begin{gathered}
F_{k}=\left\{f_{\mathbf{j i}}(0): \mathbf{j} \in \Lambda_{k}\right\} \cap B\left(\rho^{k}\right), \\
\hat{F}_{k}=\left\{\mathbf{u}=\left(\rho_{\mathbf{j}} M_{\mathbf{j}}, f_{\mathbf{j}}(0), k\right): f_{\mathbf{j i}}(0) \in F_{k}\right\} .
\end{gathered}
$$

Then

$$
\# \hat{F}_{k} \geq \# F_{k} .
$$


Note that for any $\mathbf{u}=\left(\rho_{\mathbf{j}} M_{\mathbf{j}}, f_{\mathbf{j}}(0), k\right) \in \Gamma_{k}$, by (2.7) we have

$$
\# O(\mathbf{u})=\# O(\mathbf{v}) \text { for every } O(\mathbf{v}) \in[O(\mathbf{u})] .
$$

Hence by the finite type of the IFS we have

$$
M=\sup \left\{\# O(\mathbf{u}): \mathbf{u} \in \Gamma_{k} \text { and } k \in \mathbb{N}\right\}<\infty .
$$

We will complete the proof of our theorem by demonstrating the following proposition.

Proposition. $\# F_{k} \leq M 4^{d} \delta^{-d}$.

We first show:

Lemma 2. There exists a sequence $\left\{\hat{G}_{k}\right\}, \hat{G}_{k} \subset \hat{F}_{k}$, such that $\# \hat{G}_{k} \geq \# \hat{F}_{k} / M$ and the family $\hat{G}_{k}(O)=\left\{f_{\mathbf{u}}(O): \mathbf{u} \in \hat{G}_{k}\right\}$, where $f_{\mathbf{u}}$ is defined by (2.5), is disjoint.

Proof. First take any $\mathbf{u}_{1} \in \hat{F}_{k}$ and consider the set

$$
F\left(\mathbf{u}_{1}\right)=\left\{\mathbf{u} \in \hat{F}_{k}: f_{\mathbf{u}}(O) \cap f_{\mathbf{u}_{1}}(O) \neq \emptyset\right\} .
$$

Then take $\mathbf{u}_{2} \in \hat{F}_{k} \backslash F\left(\mathbf{u}_{1}\right)$ and consider the set

$$
F\left(\mathbf{u}_{2}\right)=\left\{\mathbf{u} \in \hat{F}_{k}: f_{\mathbf{u}}(O) \cap f_{\mathbf{u}_{2}}(O) \neq \emptyset\right\} .
$$

Now take $\mathbf{u}_{3} \in \hat{F}_{k} \backslash\left[F\left(\mathbf{u}_{1}\right) \cup F\left(\mathbf{u}_{2}\right)\right]$, and so on. Continuing this process until

$$
\hat{F}_{k} \backslash\left[F\left(\mathbf{u}_{1}\right) \cup \ldots \cup F\left(\mathbf{u}_{k}\right)\right]=\emptyset
$$

we obtain a set

$$
\hat{G}_{k}=\left\{\mathbf{u}_{1}, \ldots, \mathbf{u}_{k}\right\} \subset \hat{F}_{k} .
$$

From (3.3) we have $\# \hat{G}_{k} \geq \# \hat{F}_{k} / M$. By definition $\hat{G}_{k}(O)=\left\{f_{\mathbf{u}}(O): \mathbf{u} \in \hat{G}_{k}\right\}$ is disjoint, and consequently the lemma is proved.

Now we are able to complete the proof of the proposition. By Lemmas 1 and 2 there exists a family of disjoint balls

$$
\mathbb{B}_{k}=\left\{B\left(f_{\mathbf{u}}\left(x_{0}\right), \delta \rho^{k}\right): \mathbf{u}=\left(\rho_{\mathbf{j}} M_{\mathbf{j}}, f_{\mathbf{j}}(0), k\right) \in \hat{G}_{k}\right\}
$$

such that

$$
B\left(f_{\mathbf{u}}\left(x_{0}\right), \delta \rho^{k}\right) \subset f_{\mathbf{u}}(O) \text { for any } \mathbf{u} \in \hat{G}_{k} .
$$

Observe that $\# \mathbb{B}_{k}=\# \hat{G}_{k}$. By (3.1) and (2.3) for any $\mathbf{u}=\left(\rho_{\mathbf{j}} M_{\mathbf{j}}, f_{\mathbf{j}}(0), k\right) \in \hat{G}_{k}$ we have

$$
\begin{aligned}
\left\|f_{\mathbf{u}}(O)-f_{\mathbf{j i}}(0)\right\| & :=\sup \left\{\left\|x-f_{\mathbf{j i}}(0)\right\|: x \in f_{\mathbf{u}}(O)\right\} \\
& \leq \sup \left\{\left\|x-f_{\mathbf{j}}(0)\right\|: x \in f_{\mathbf{u}}(O)\right\}+\left\|f_{\mathbf{j}}(0)-f_{\mathbf{j i}}(0)\right\| \\
& \leq \operatorname{diam} \rho_{\mathbf{j}} M_{\mathbf{j}}(O)+\rho_{\mathbf{j}}\left\|0-f_{\mathbf{i}}(0)\right\| \\
& \leq \rho_{\mathbf{j}} \operatorname{diam} O+\rho_{\mathbf{j}}\left\|f_{\mathbf{i}}(0)\right\| \leq 2 \rho_{\mathbf{j}} \leq 2 \rho^{k}
\end{aligned}
$$

It follows that

$$
\begin{aligned}
\operatorname{diam} \bigcup \mathbb{B}_{k} & \leq \operatorname{diam}\left(F_{k} \cup \bigcup \mathbb{B}_{k}\right) \\
& \leq \operatorname{diam}\left(F_{k} \cup\left\{f_{\mathbf{u}}(O): \mathbf{u} \in \hat{G}_{k}\right\}\right) \\
& \leq 2 \rho^{k}+2 \rho^{k}=4 \rho^{k} .
\end{aligned}
$$


Thus $\bigcup \mathbb{B}_{k}$ is contained in a ball $B\left(4 \rho^{k}\right)$ of radius $4 \rho^{k}$ in $\mathbb{R}^{d}$. Since $\mathbb{B}_{k}$ consists of disjoint balls we have

$$
\sum\left\{V(B): B \in \mathbb{B}_{k}\right\} \leq V\left(B\left(4 \rho^{k}\right)\right)
$$

where $V(B)$ denotes the volume of a ball $B$ in $\mathbb{R}^{d}$. Observe that the volume of an $r$-ball $B$ in $\mathbb{R}^{d}$ is $V(B)=C_{d} r^{d}$, where $C_{d}$ is a constant depending only on $d$. (Note: $C_{1}=2$ and $\left.C_{2}=\pi !\right)$ Since $\# \mathbb{B}_{k}=\# \hat{G}_{k}$ we have

$$
\# \hat{G}_{k} C_{d}\left(\delta \rho^{k}\right)^{d} \leq C_{d}\left(4 \rho^{k}\right)^{d} .
$$

Consequently from (3.2) and from Lemma 2 we get

$$
\# F_{k} \leq \# \hat{F}_{k} \leq M \# \hat{G}_{k} \leq M 4^{d} \delta^{-d} .
$$

Thus the Proposition, and hence the Theorem, is proved.

\section{ACKNOWLEDGEMENTS}

I am grateful to the referee for his (her) suggestions for improving the exposition of this paper. I also want to thank T. Hu and S. Ngai for many valuable discussions during the preparation of this paper.

\section{REFERENCES}

[F] K. Falconer, Fractal Geometry, Mathematical Foundations and Applications, Wiley 1990. MR 92j:28008

[H] J. E. Hutchinson, Fractals and self-similarity, Indiana Univ. Math. J. 30(1981), 713-747. MR 82h:49026

[L] S.P. Lalley, $\beta$-expansions with deleted digits for Pisot numbers, Trans. Amer. Math. Soc. 349(1997), 4355-4365. MR 98f:11084

[LN] K. Lau and S. Ngai, Multifractal measure and a weak separation condition, Adv. in Math. 141(1999), 45-96. MR 2000d:28010

[NW] S. Ngai and Y. Wang, Hausdorff dimension of self-similar sets with overlaps, J. London Math. Soc., to appear.

[Z] M.P.W. Zerner, Weak separation property for self-similar sets, Proc. Amer. Math. Soc. 124(1996), 3529-3539. MR 97c:54035

Department of Mathematical Sciences, New Mexico State University, Las Cruces, New Mexico 88003-8001 and Institute of Mathematics, P.O. Box 631, Bo Ho, Hanoi, VIETNAM

E-mail address: nnguyen@nmsu.edu 